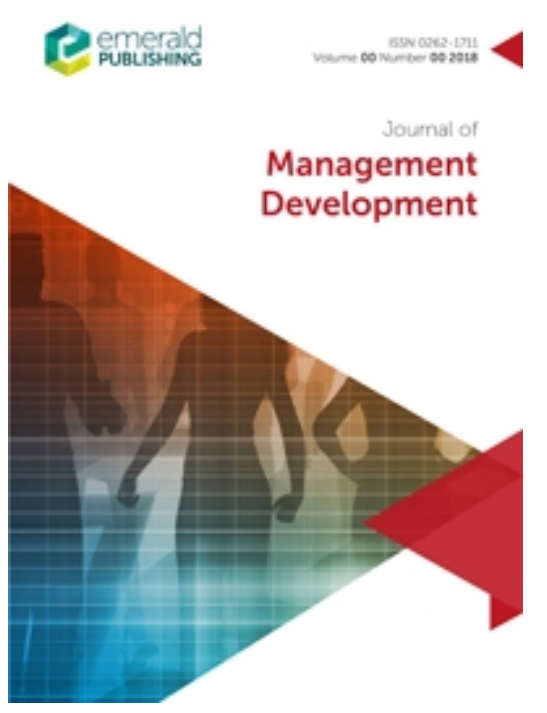

\title{
Toward Better Understanding Developmental Reflection Differences for Use in Management Development Research and Practice
}

\begin{tabular}{|r|l|}
\hline Journal: & Journal of Management Development \\
\hline Manuscript ID & JMD-11-2020-0349 \\
\hline Manuscript Type: & Original Article \\
\hline Keywords: & $\begin{array}{l}\text { Experiential learning, Individual development, Leadership development, } \\
\text { Management Development, Post-experience learning }\end{array}$ \\
\hline \multicolumn{2}{|l}{} \\
\end{tabular}

\section{SCHOLARONE ${ }^{m}$ Manuscripts}




\section{Structured Abstract}

Purpose: We introduce a new approach to developmental reflection in which the focus is on differences in how people reflect. When reflecting on challenging experiences, people achieve better development when they tend to look for causes of what happened within changeable personal characteristics and they subsequently focus on improvement of those personal characteristics.

Design/methodology/approach: Supervisors and subordinates with leadership responsibilities in diverse jobs in varied industries provided survey data (444 individuals in a psychometric testing sample, and 419 paired subordinate/supervisor dyads in a model-testing sample).

Findings: The reflection difference construct had the expected factor structure, reliability, and was distinguishable from eight conceptually related variables in the literature. Reflection differences were predicted by theoretically-relevant job, person, and situational variables and were associated with development and performance outcomes.

Originality: This study establishes support for: a) the new theoretical framing of reflection differences, b) a new preliminary model of antecedents and outcomes, and c) an initial scale for future research and practice that can be more explicit about understanding and addressing underlying differences in how people reflect.

Practical Implications: The reflection construct might be used for prediction to identify which individuals are likely to get the most from challenging experiences and improve. Further, by identifying predictors of reflection, ideas for enhancing reflection are provided. Also, by uncovering specific underlying dimensionality of reflection, this offers specific targets for interventions beyond generally encouraging people to reflect.

Article Classification: Research Paper; Keywords: Experiential learning, Individual development, Leadership development, Management Development, Post-experience learning 
Toward Better Understanding Developmental Reflection Differences

for Use in Management Development Research and Practice

Reflection is an important, impactful, and ubiquitous process linking various experiences to learning and development outcomes. Previous research suggests that learning occurs as individuals go through challenging experiences, reflect, and plan for the future (e.g. Dewey, 1938; Knowles, 1975; Kolb, 1984; Marsick \& Watkins, 1990; Rogers, 1969; Smith, 2001); however, Daudelin (1996) cautioned that, "Even though reflection has been an important part of traditional educational experiences since ancient times, its power is just beginning to be harnessed as a deliberate tool of managerial learning" (p. 39). Likewise, McCall (2010; p. 3) noted that "using experience effectively to develop leadership talent is a lot more complicated and difficult than it appears to be" (p. 3). Calls for more attention to this area of research in relation to managerial and leader development are common (cf. Avolio, 2004; DeRue \& Wellman, 2009; Zaccaro et al., 2000). Still, reflection as a construct has not been extensively examined, whether in terms of which types or parts of reflection are most effective, or which types of people or situations tend to produce reflections that are most developmentally productive. There is a need to better understand and manage the reflection construct as a bridge between experience and development.

In the present article, we expand understanding by identifying two key dimensions of reflection that are crucial to advancing both theory and practice. We show that these dimensions of reflection are distinct from conceptually-related constructs, that the differences are predicted by important person, job and organizational variables, and that they predict important development and career outcomes. We integrate all of these variables into a preliminary model of reflection differences. Overall, we attempt to advance understanding of reflection by moving the focus of reflection from a practice- or interventionbased perspective, in which tactics and techniques are applied to produce reflection and outcomes, to a framework that involves measurable differences. 
DEVELOPMENTAL REFLECTION DIFFERENCES

\section{Developmental Reflection Differences as a Bridge Between Experience and Development}

According to a variety of theoretical perspectives and studies in the literature, learning occurs when individuals encounter challenging or atypical experiences and then reflect on them (DeRue \& Wellman, 2009; Dewey, 1938; Knowles, 1975; Kolb, 1984; Marsick \& Watkins, 1990; Olivares, Peterson, \& Hess, 2007; Rogers, 1969; Smith, 2001). In practice, this can be employed when reflection is prompted or encouraged. For example, Ellis and Davidi (2005) addressed "after-event reviews" (AERs) in which participants are typically asked to analyze their past performance or behavior and to suggest explanations for their successful and/or failed actions. Generally, this type of "systematic reflection" has been shown to be a productive influence on performance (Ellis, Carette, Anseel, \& Lievens, 2014). Likewise, the US Army's After Action Review (AAR) is an intervention, similar to AERs, designed to maximize learning from an event (Salter \& Klein, 2007). Coached reflection, similarly, is a structured event in which the learner is given tools to think through an experience in order to identify what they learned from it (cf. Cox, 2005; Daudelin, 1996; Quinton \& Smallbone, 2010). Such processes can thus be a valuable tool in efforts to enhance development.

However, reflection does not necessarily need to be prompted, coached, or guided. Such processes might emerge from within the individual. In fact, while work described above has focused on inducing reflection via structured guidance, other literature has implied that reflection might occur as a natural process, or at least spontaneously during or after a developmental experience (Schon, 1983). The actual frequency or depth of this process, however, can differ notably between individuals. Smith (2001) suggested that reflection does not come naturally to many people. Conversely, there may be differences in how adept people are at reflection and also the degree to which they are predisposed to exhibiting reflection behaviors. This poses an interesting question: Might a tendency to engage in reflection behavior be a key difference among people? 
Some recent exploratory data offer support to the notion that there could be individual differences in how people reflect. In a qualitative study (Maurer, Leheta, \& Conklin, 2017), participants reported some reflection connected with important work-related events that ranged from "a little reflection during" to "a lot of reflection long after" and there were considerable self-reported differences in reflection content. Some participants spent time simply replaying the experience in their minds, while others pursued an underlying understanding of what occurred. These initial qualitative findings suggest that developmental reflection behaviors will differ from person to person, and the findings point to a need to better comprehend the nature of individual reflection differences; however, research thus far has not systematically and empirically studied developmental reflection as a key differentiator across people in relation to development.

\section{What Dimensions of Reflection Lead to Development from Experience?}

Models of behavior featuring two types of processing (such as effortful vs. automatic or slower vs. faster processes; see Chaiken \& Trope, 1999; Smith \& DeCoster, 2000; Evans, 2003; Strack \& Deutsch, 2004) suggest that deeper processing results in new connections and behavioral schemata that drive novel behavior, while shallower processing may simply (re)activate existing schemas and behavioral implications (cf. Strack \& Deutsch, 2004), or potentially even biases. Deep analytical processing represents the amount of concerted cognitive effort invested in exploring, understanding, and then modifying or radically changing one's mental models based on a challenging experience.

Along these lines, Hatten and Smith (1995) differentiated written reflections ranging from merely recounting with no attempt at reasoning, through increasing levels of analytic thinking with greater explaining, hypothesizing, and critical reflection. Anseel, Lievens and Schollaert (2009) suggest that effortful, controlled and intentional focus and thinking can produce more meaningful change in mental models, attitudes, and behavior (Anseel et al., 2009; Ellis \& Davidi, 2005). Consistent with this literature, Ellis, Mendel and Nir (2006) suggested that interventions that target thinking about prior performance 


\section{DEVELOPMENTAL REFLECTION DIFFERENCES}

such as After Event Reviews promote learners' performance by focusing them on analyzing their experience in terms of internal and specific causes as opposed to external and general ones.

Thus, the literature on reflection and related behavior suggests surface or shallow reflection that might involve simply reliving or repeating an experience without regard to an understanding and deeper causal analysis seems less productive according to this literature. Importantly, framing reflection as analytically focused on one's own influence and possible areas of change is aligned with employee and leader development in which the focus is on making positive, constructive developmental change in skills, knowledge, or competencies (cf. Noe, Wilk, Mullen \& Wanek, 1997). We posit reflection in a manner that helps integrate the literature on reflection with the literature on employee and leader development. That is, there is a focus on understanding those causes of challenging experiences that are under the control of the actor, and understanding of those causes leads to a motivated plan toward developing and improving those causal factors that contributed to the experience.

To the extent that reflection focuses on changeable or improvable factors (e.g. behaviors, skills, knowledge, etc.) reflection is conducive to development. Given this discussion of literature on cognitive processing and reflection, we identify a key underlying dimension of developmental reflection to be a causal analysis of changeable person characteristics (abbreviated "CA"). That is, when people reflect on an experience, they analyze the personal causes of the experience that are changeable, such as their own behavior, effort, knowledge, skills, or affect/emotion-related constructs.

This leads to the discussion of a second underlying dimension of reflection that links experiences to development - the cognitions related to actions the individual can take to improve. Hatten and Smith (1995, p. 40) defined reflection as "deliberate thinking about action with a view to its improvement." Inherent in this definition is the centrality of positive action to the process of reflection; reflection should include a plan to improve the outcomes of the situation or perform better in the future. For instance, Gray's (2007) definition of reflection emphasizes the need to 
balance thinking with action, otherwise reflection will only lead to abstract theorizing. A focus on one's own characteristics and behaviors with an eye toward future improvement and goals is a psychological process that exists in literature on self-systems (Strauss, Griffin, \& Parker, 2012; Markus \& Nurius, 1986; Markus \& Wurf, 1987). Likewise, having a future-oriented approach in defining one's self and one's goals has been found to "stretch individuals' aspirations and broaden their creative thinking about future possibilities" (Strauss et al., 2012, p. 581). This makes them more motivated and proactive in self-development and learning from their experiences by using these experiences and the lessons learned to control and direct their own future behaviors (Oyserman \& Markus, 1990, Hoyle \& Sherill, 2006; Markus \& Nurius, 1986; Strauss et al., 2012).

This process of identifying future action implications that flow from identifying the causal factors that one can control (e.g. one's own behaviors, skills, knowledge, effort, emotions, etc.) is a critical part of developmental reflection. Such behavioral schemata are more developmentally constructive as a result of deeper and more analytical processing. Developmental Action Implications (abbreviated "DAI") is thus another key underlying dimension of reflection in that people identify, via their analysis of experiences and underlying causes, the implications for positive change in the causal factors identified — the plan or intentions that can lead to enhancement and improvement. That is, when people reflect on an experience and analyze the causes of the experience (i.e. causal analysis - $C A$-- of the changeable person characteristics), then implications for developmental action will naturally emerge from those insights in the form of development implications for the changeable person characteristics.

Thus, as described above, we propose a new way to theoretically frame the underlying dimensionality of developmental reflection as a process of causal analysis and emergent action implications. Together, the $C A$ and $D A I$ reflection processes form the bridge between challenging developmental experiences and the developmental behaviors or actions that lead to positive change in skill that follows experience and reflection. The logic of these two underlying dimensions implies a sequential 
relationship between them in which CA naturally leads to emergent developmental action implications (DAI) for improving the characteristics analyzed in CA.

Based on this conceptualization of proposed overall reflection processes, we next place this construct within a network of predictors and outcomes that further illustrates the nature of the construct and its relevance within the literature. The central theoretical predictions are illustrated in Figure 1, that is, job (job challenge), individual (learning goal orientation), and situational (error management) predictors elicit greater causal analysis of underlying changeable person characteristics in reflection (e.g. CA) and this focus on causal analysis naturally leads to developmental action implications regarding improvement of the same underlying changeable characteristics (DAI). These improvement implications then lead to developmental improvement which leads to performance and success. We elaborate on the model hypotheses regarding predictors and outcomes of reflection differences below.

\section{Insert Figure 1 about here}

\section{What Kind of Occupational, Individual and Situational Variables Predict Reflection Differences?}

While a number of predictors of reflection could exist, to control size and complexity in creating a preliminary model, highly theoretically relevant, proximal, and salient predictors were chosen as exemplar variables from each category.

Occupational Antecedent: Challenging Experiences on the Job. According to the literature described above, having a greater amount of challenging experiences should lead to greater reflection on challenging experience and having fewer experiences will be associated with less reflection. Having challenging, novel, or otherwise important experiences stimulates more reflection. We thus argue that the presence of challenging experiences will be associated with higher levels of causal analysis and also emergent developmental action implications in the reflection process. Therefore, we posit:

Hypothesis 1: Challenging experiences arising from one's occupation will be positively associated with developmental reflection. 
Individual Antecedent: Learning Goal Orientation. Managers high in learning goal

orientations gain more from developmental assignments (Dragoni, Tesluk, Russell \& Oh, 2009);

furthermore, individuals with high learning goal orientations tend to engage in more thinking about their own thought processes during training experiences (e.g., Schmidt \& Ford, 2003).

While this work suggests links between goal orientation and development, the processes and constructs underlying the links have not been examined to any significant extent. In the current study, it seems worthwhile to test whether the links of learning goal orientation to development occur through increased reflection processes. We argue that a strong motive to learn might serve as a driver of reflection that involves trying to learn from the experience and grow one's relevant skills (Ellis et al., 2006).

Suggesting promise for that idea, Anseel et al. (2009) demonstrated that learning goal orientation is related to measures of word count as indicators of depth of processing during reflection. Extending this prior research into the present framework, it seems likely that individuals who strive to learn the most that they can from experiences (high learning goal orientation) will be more likely to think deeply and to a greater extent (Anseel et al., 2009; Schmidt \& Ford, 2003), to analyze controllable causal factors of challenging experiences (due to one's attributes and behavior), and (ultimately, and via one's stronger focus on learning) identify ways to improve one's attributes/behaviors or learn from the experiences - thus, exhibiting higher developmental reflection tendency, characterized by higher levels of CA (and resulting DAI).

Hypothesis 2: Learning goal orientation will be positively associated with developmental reflection.

Organizational Antecedent: Organizational Error Management. There is only a limited amount of research on reflection behavior in work settings, and there is even less on the environmental conditions that help enhance or produce this behavior. According to Seibert (1999), "there is a particular need for 
DEVELOPMENTAL REFLECTION DIFFERENCES

information on the way in which the immediate work environment within which such experiences unfold influences managers' reflective capacities" (p. 54).

Some learning takes place when people are encouraged to learn from errors or mistakes they have made (Heimbeck, Frese, Sonnentag, \& Keith, 2003) and when they think about errors (e.g., planning, monitoring, and evaluating one's actions) (Keith \& Frese, 2005). There are organizational contexts that actually recognize the value of learning from errors and that promote development and learning from them (van Dyck, Frese, Baer \& Sonnentag, 2005). It seems likely from the present discussion that developmental reflection could very well be positively influenced by an error management culture that does not punish errors but instead encourages dealing with errors constructively and learning from them. People in such work settings may be more likely to analyze difficult experiences they have at work and to examine their own behavior and their own characteristics (e.g. skills, knowledge) as potential causes of the challenges they experience within such a setting, and they may be more likely to develop plans of action to ameliorate those sources of difficulty.

Hypothesis 3: The presence of organizational error management will be positively associated with developmental reflection.

\section{Do Reflection Differences Predict Development and Success?}

Development and skill improvement are important for a variety of reasons. From the perspective of human capital theory, employees invest in their own human capital (Becker, 1975) and those who invest more time, effort, training, and experience should reap rewards of such investments. Employees with a higher personal investment in and dedication to development could reap a greater degree of rewards from their efforts including more favorable career outcomes (cf. Boudreau, Boswell, \& Judge, 2001). With respect to reflection, those who spend time analyzing and thinking strategically about the next developmental course of action that would be most appropriate to enhance their effectiveness are going to be more likely to be successful at development. This suggests a link between developmental reflection and 
subsequent development. The present model explicitly ties developmental reflection to leader and employee development, something that has not been done in prior research, but which has great potential to advance this area of literature.

Hypothesis 4: Developmental reflection is expected to be positively related to subsequent development/improvement.

Hypothesis 5: Development will be positively associated with a) performance and b) career success.

\section{Method}

\section{Procedure Overview}

The study involved two main goals. First, we demonstrated that the reflection construct is indeed distinct and offers unique value beyond existing constructs of some conceptual similarity. We did this by including a significant number of conceptually related constructs for comparison and control, both testing for relationships with the reflection construct as well differentiating and distinguishing the comparator constructs from the reflection construct. The second goal involved testing the proposed model of developmental reflection including both antecedents as well as proximal and distal outcomes of reflection. As a conservative test of the new measures we controlled for the comparator constructs in the model.

Participants were recruited through two professional research participant recruiting organizations, Qualtrics and StudyResponse.com. Having data from two recruiting organizations served to help diversify our sample. The data received via these services were combined to create the two samples described below. Respondents were asked to help recruit supervisors who independently completed a supervisor survey. Participants who did not recruit paired supervisors were utilized for the psychometric testing part of our model (Sample 1). Participants with supervisors that participated were used to test the hypotheses of this study (Sample 2). In return for their participation, subordinate respondents were offered $\$ 20$ gift cards while supervisory respondents, who completed a much shorter survey, were provided with $\$ 10$. All 


\section{DEVELOPMENTAL REFLECTION DIFFERENCES}

surveys were completed online. Both subordinates and supervisors provided demographic information, while subordinates provided information on individual differences, job and organizational variables, reflection variables, and career outcomes. Supervisors rated their subordinates' development and performance.

\section{Participants}

Participants were initially identified by the recruiting organizations as eligible for the study (i.e. were part of the US workforce, employed full time (at least 30 hours per week) with at least 1 year of work experience, at least 18 years of age, fluent in English, and had worked with a current supervisor for at least the last 12 months. We focused on managerial and leadership skill development and performance and therefore an additional eligibility requirement was that a respondent had either supervisory responsibilities or some informal leadership activities as part of his/her job. Across recruiting organizations, we obtained 444 individual responses for Sample 1, and 419 paired subordinate/supervisor dyads for Sample 2. In terms of demographics, Sample 1 was $42 \%$ female and $70.3 \%$ Caucasian. Most respondents (51\%) had attained a college degree, with the remaining reporting a lower (20\%) or higher (29\%) level of education. Most participants (55\%) held a mid-level position in their organization. Sample 2 was $38 \%$ female and $75.6 \%$ Caucasian. Most respondents (61\%) had attained a college degree, with the remaining reporting a lower $(10 \%)$ or higher $(30 \%)$ level of education. Most participants $(59 \%)$ held a mid-level position in their organization. For both samples, respondents were diverse in terms of job type, including clerical, management, engineering, sales, information technology, and research and development.

\section{Measures}

The measures in the present study were in three categories: a) the predictor variables, including job challenge, organizational error management, and learning goal orientation; b) the measures of developmental reflection (causal analysis and developmental action implications) along with a number of comparator measures. That is, to ensure empirical integrity of the reflection scales, we took a 
relatively rigorous and conservative analytic approach of examining not only the factor structure and reliability of the reflection measures, but also their distinctness and uniqueness relative to a number of other, existing comparator variables in the literature. We collected a number of scales that were conceptually related in some way to our proposed construct. Our goal was to choose variables with possible risk of being empirically redundant. We examined the independent value of the new construct/measure by confirmatory factor analysis and by including the comparators as control variables in modeling of the new scales. A literature review revealed eight scales that seemed generally related to the idea of developmental reflection via a tendency to think about oneself and one's circumstances (e.g. need for cognition, mindfulness, rumination and reflection in private selfconsciousness) and/or being open to the possibility of change and influence in one's experiences (e.g. openness, implicit theory of ability, work locus of control, and resistance to change). The third category of measures included: c) development and career outcomes of developmental reflection, including development, performance, and success (promotions). The details of all three categories of measures are given in the appendix for an interested reader, along with detailed confirmatory factor analyses (CFA) showing the expected dimensions of reflection differences, reliability of the scales, and their distinctiveness from the other related (comparator) constructs.

\section{Results}

Zero order correlations between the reflection and comparator scales (see Table 1) show some degree of positive correlations between the reflection measures and the comparator variables as expected given that they were chosen due to their conceptual similarity. However, none of the correlations are so high as to indicate that they represent the same variable, similar to the conclusions from the CFA analyses of the measures. In total then, our analyses suggest that the reflection measures are correlated with the existing comparator construct measures as should be expected, but they are also distinct constructs. 


\section{DEVELOPMENTAL REFLECTION DIFFERENCES}

We conducted a formal path model analysis to test the proposed model and to demonstrate the association of reflection with the individual, occupational, and organizational variables, the association of reflection with development, and the ultimate effects on performance and career outcomes. All proposed hypotheses were tested simultaneously in a path model. While the fully-mediated chain shown in Figure 1 is most central to the theory, for completeness we also examined partial mediation in all path effects from predictors, such that the preceding variables in the causal chain predicted both their mediator and outcomes. Therefore, we also estimated the parallel effects from predictors to DAI, from CA to development, and from DAI to performance and promotions. Demographic variables (age, gender, race, and education) were used as controls predicting all variables in the model.

Insert Tables 1, 2 and 3 about here

Table 2 shows the means, standard deviations, and intercorrelations among the variables in this model, collected in Sample 2. Tables 3a and $3 b$ illustrate the results of the two path models testing these effects, and path results are also presented in Figure 1. Overall, the model fit the data acceptably well $\left(\chi^{2}=\right.$ 120.93, df. $=11 ; \mathrm{RMSEA}=.16 ; \mathrm{CFI}=.92 ; \mathrm{SRMR}=.06$.). An alternative model with only direct effects included (that is, only antecedents to CA, CA to DAI, DAI to development, and development to performance and career success $)$ fit the data poorly $(\mathrm{CFI}=.85 ; \mathrm{RMSEA}=.17 ; \mathrm{SRMR}=.10)$ suggesting the parallel effects are worth examining, which we do below.

In terms of the central predictions involving antecedents of reflection, job challenge was indeed found to predict $\mathrm{CA}(\mathrm{b}=.22, \mathrm{p}<.01)$, as did learning goal orientation $(\mathrm{b}=.30, \mathrm{p}<.01)$ and error management culture $(b=.25, \mathrm{p}<.01)$. Hypotheses 1,2 and 3 were thus fully supported. As expected, given the theoretical relationship between $\mathrm{CA}$ and DAI as part of reflection, CA predicted DAI $(b=.57, \mathrm{p}$ $<.01)$ in the model. With regard to the proximal and distal outcomes of reflection, DAI predicted supervisor-rated development $(\mathrm{b}=.80, \mathrm{p}<.01)$. Supervisor-related development predicted both subordinate performance $(b=.58, \mathrm{p}<.01)$ and career success $(\mathrm{b}=.10, \mathrm{p}<.01)$. Hypotheses 4 and 5 were 
thus fully supported. In terms of parallel path estimates alongside the central effects, all three antecedent variables also predicted DAI in addition to $\mathrm{CA}$, although these coefficients were lower than those to CA in each instance. This suggests that the antecedents may play a role in DAI directly and not just through CA. In terms of effects on development, the path from CA was not significant suggesting that CA did not directly affect development once DAI was accounted for. With respect to effects on outcomes, DAI had effects directly on promotions and performance, suggesting that DAI plays an ongoing role in performance and promotion beyond that accounted for by development.

Indirect effects for the model are summarized in Table 4. In terms of the substantive effects, the antecedent variables were found to be positively associated with career success and supervisor-rated performance through CA, DAI, and supervisor development ratings (all estimates significant, and no confidence intervals for these effects included zero). CA was found to be significantly associated with both career success and supervisor rated-performance through DAI and supervisor-rated development, while DAI was associated with career success and supervisor-rated performance through supervisor-rated development.

\section{Insert Table 4 about here}

To examine the robustness of our model, we reran the hypothesized model presented above by including comparator variables. The comparator variables were used in establishing the psychometric properties of our proposed scale in Sample 1 by examining distinctiveness, but they were not central to our theory and proposed model; however, by collecting them in Sample 2 they can be used to test the robustness of the hypothesized model including the antecedents. We tested these robustness models by including one comparator at a time and also by including all comparators at once in which the comparators were used as control variables alongside the demographics. In these very conservative tests of robustness, the models thus controlled for mindfulness, work locus of control, openness, reflection-private self, 


\section{DEVELOPMENTAL REFLECTION DIFFERENCES}

rumination-private self, need for cognition, and resistance to change. None of the hypothesized model path coefficients sign or significance changed, and their magnitude was substantively also unchanged.

\section{Discussion}

Prior literature has called for more research on the ways underlying process variables explain how experience translates into development, and why some employees respond well developmentally to challenges and others do not. Much prior research related to reflection treats reflection as part of an intervention to help people draw value out of experience (e.g. AERs, AARs, coached reflection). In the present article, a review of the literature on reflection and cognition identified two underlying dimensions of developmental reflection on which employees may differ, helping to explain differences in development. By analyzing causes of challenge as originating in changeable person characteristics and identifying emergent developmental action implications, employees can make the required transition from difficult and challenging experience into actionable improvement. Those who engage in this process will be more likely to create developmental value.

Enhancing confidence in this framework via careful empirical tests, the dimensions of reflection offered were distinguishable from eight conceptually-related comparator scales. They were also featured in a preliminary model of reflection differences that included theoretically-relevant person, job and organizational variables used as predictors, and development, performance, and career variables as outcomes of reflection differences. By placing the developmental reflection construct at the center of this preliminary model, we further demonstrated construct validity and also the usefulness of differences in reflection for predicting development. The model suggested that those who are more learning oriented (Vandewalle \& Cummins, 1997), have more challenging jobs (McCauley et al., 1994), and work in organizational contexts that are higher in error management (van Dyck et al., 2005) will exhibit greater developmental reflection. Those who show greater developmental reflection will demonstrate greater development, and subsequently, higher performance and success. Given the direct and indirect effects 
observed, it appears that both dimensions of developmental reflection differences can help to explain differences in development and success that may result from experience.

\section{Originality of the Findings}

Three dimensions that underlie a theoretical contribution — originality, utility, and prescience (Corley \& Gioia, 2011) — are reflected in the present article. In terms of originality, the present article moves the theoretical focus of reflection from a practice- or intervention-based perspective in which tactics and techniques are applied to produce reflection and outcomes to a framework in which developmental reflection is a difference construct. Developmental reflection as a differentiating behavioral variable presented here might be used within a number of streams of future research. The present model provides a contribution in the form of theory-building through its development of the reflection construct, its identification of dimensions of reflection, and its exploration of relationships with predictors and outcomes (Colquitt \& Zapata-Phelan, 2007). The constructs proposed and tested provide an opportunity for theory expansion, contributing to the literature at the highest level in Colquitt and Zapata-Phelan's (2007) taxonomy of theoretical contributions.

\section{Utility of the Findings}

In terms of the utility that underlies a theoretical contribution, Corley and Gioia (2011) argue that practical utility is valuable in considering theoretical contributions, and so some consideration of possible "applied research" is useful here. Applied research based on the present model could fall into two categories: individual difference predictive studies and experimental research in which manipulations are used. For individual difference studies, here we showed that differences seem to exist and relate to constructs and outcomes that matter in the organizational literature, and we provided initial measures - all of which are valuable preconditions to investing in future longitudinal research along these lines. The present study sets out a sound theoretical model to help guide future research on the construct as a difference variable. The reflection measure provides contributions to the field as a resource 


\section{DEVELOPMENTAL REFLECTION DIFFERENCES}

to use in future research (and possibly practice). The construct could be used as a predictor of performance, motivation, development, and adaptability in training and other field settings. To the extent that the construct predicts effectiveness in adapting to leadership and other challenging roles and learning and developing necessary skills and success, it might be considered for use in selection and placement models. The construct might be used to identify individuals who will get the most from challenging leadership experiences and who are most likely to pursue development and improvement of leadership characteristics in themselves following challenging assignments. The difference construct might be tested for relationships with important behavioral and performance criteria in other practical settings.

Future research could also be done on ways of influencing this construct in personnel to improve learning and development, as well as performance and success. By identifying the specific underlying dimensionality that is key to reflection, the present study provides a more precise target for reflection interventions in the future. That is, instead of more generally instructing people to reflect on prior behavior, they might be coached to focus specifically on changeable, internal characteristics that caused outcomes in performance and then to consider the developmental improvement implications that flow from those new insights. Via such targeted reflection interventions, developmental effectiveness of reflection exercises might be enhanced. Research could test these ideas. Further, the individual difference as well as the situational predictor constructs in the model could provide clues for improving reflection. Enhancing learning goals or error management in a given situation might produce greater developmental reflection. The present findings suggest that reflection differences may be influenced by person, job and organizational context variables, thus providing some interesting questions about how malleable the construct is. While prior literature has examined interventions to influence reflection, it has not done so within the framework presented here. Future research might take this reflection difference framework and examine possible influence tactics as well as conduct test-retest studies to further understand the nature of this construct. 


\section{DEVELOPMENTAL REFLECTION DIFFERENCES}

\section{Prescience of the Findings}

In terms of prescience, the present framework offers additional insight into a construct that rests at the nexus of several streams of research including experiential learning and education, training, leadership development, organizational culture, and motivation. Further, such a construct has the potential to play a key role in practical concerns such as training, development, selection, and placement. All of these areas of research and practice are likely to continue to be important in the future and therefore the present framework has the potential to contribute to several key areas.

\section{Conclusion}

The present study had several strengths including data from multiple sources (respondents and their supervisors), within diverse jobs and industries, obtained from two separate data recruiting organizations, exploring a significant variety of comparator and control variables, and several exemplar predictors that were specifically chosen for their theoretical relevance. To control/reduce model complexity, we chose theoretically and established examples of constructs from individual, job, and situational realms to create the initial model; however, this model could be elaborated or changed in the future. As an important step in the literature, it provides a good way to frame these reflection differences and show some initial relationships with highly relevant constructs from organizational behavior. In addition, the present data were not longitudinal and it would be useful to explore the predictors, reflection, and development variables over time. Finally, the measures of reflection could be elaborated or improved with more detail or to target specific types of skills. The present research demonstrated that there is empirical value in framing reflection differences in this type of overall framework. 
DEVELOPMENTAL REFLECTION DIFFERENCES

\section{References}

Anseel, F., Lievens, F., \& Schollaert, E. (2009). Reflection as a strategy to enhance task performance after feedback. Organizational Behavior and Human Decision Processes, 110, 23-35.

Avolio, B. J. (2004). Examining the full range model of leadership: Looking back to transform forward. In D. A. Day, S. J. Zaccaro, \& S. M. Halpin (Eds.), Leadership development for transforming organizations: Grow leaders for tomorrow: 71-98. Mahwah, NJ: Erlbaum.

Becker, G. S. (1975). Human capital. Chicago: University of Chicago Press.

Boudreau, J., Boswell, W., \& Judge, T. (2001). Effects of personality on executive career success in the United States and Europe. Journal of Vocational Behavior, 58, 53-81.

Cacioppo, J. T., Petty, R. E., \& Kao, C. F. (1984). The efficient assessment of need for cognition. Journal of Personality Assessment, 48, 306-307.

Chaiken, S., \& Trope, Y. (Eds.). (1999). Dual-process theories in social psychology. Guilford Press.

Colquitt, J. A., \& Zapata-Phelan, C. P. (2007). Trends in theory building and theory testing: A five-decade study of the Academy of Management Journal. Academy of Management Journal, 50, 1281-1303.

Corley, K., \& Gioia, D. (2011). Building theory about theory building: What constitutes a theoretical contribution? Academy of Management Review, 36, 12-32.

Cox, E. (2005). Adult learners learning from experience: using a reflective practice model to support work-based learning. Reflective Practice, 6, 459-472.

Daudelin, M. (1996). Learning from experience through reflection. Organizational Dynamics, 24, 36-48.

DeRue, D. S., \& Wellman, N. (2009). Developing leaders via experience: The role of developmental challenge, learning orientation, and feedback availability. Journal of Applied Psychology, 94, 859875.

Dewey, J. (1938). Experience and education. New York: Macmillan. 
Dragoni, L., Tesluk, P. E., Russell, J. E. A., \& Oh, I. (2009). Understanding managerial development: Integrating developmental assignments, learning orientation, and access to developmental opportunities in predicting managerial competencies. Academy of Management Journal, 52, 731743.

Dweck, C., Chiu, C., \& Hong, Y. (1995). Implicit theories and their role in judgments and reactions: A world from two perspectives. Psychological Inquiry, 6, 267-285.

Ellis, S., Carette, B., Anseel, F., \& Lievens, F. (2014). Systematic reflection implications for learning from failures and successes. Current Directions in Psychological Science, 23, 67-72.

Ellis, S., \& Davidi, I. (2005). After-event reviews: Drawing lessons from successful and failed experience. Journal of Applied Psychology, 90, 857-871.

Ellis, S., Mendel, R., \& Nir, M. (2006). Learning from successful and failed experience: The moderating role of kind of After-Event Review. Journal of Applied Psychology, 91, 669-680.

Evans, J. S. B. (2003). In two minds: dual-process accounts of reasoning. Trends in Cognitive Sciences, 7 , 454-459.

Gray, D. (2007). Facilitating management learning: Developing critical reflection through reflective tools. Management Learning, 38, 495-517.

Hatten, N., \& Smith, D. (1995). Reflection in teacher education: towards definition and implementation. Teaching and Teacher Education, 11, 33-49.

Heimbeck, D., Frese, M., Sonnentag, S., \& Keith, N. (2003). Integrating errors into the training process: The function of error management instructions and the role of goal orientation. Personnel Psychology, 56, 333-362.

Hoyle, R. H., \& Sherrill, M. R. (2006). Future orientation in the self-system: Possible selves, selfregulation, and behavior. Journal of Personality, 74, 1673-1696. 


\section{DEVELOPMENTAL REFLECTION DIFFERENCES}

Keith, N., \& Frese, M. (2005). Self-regulation in error management training: Emotion control and metacognition as mediators of performance effects. Journal of Applied Psychology, 90, 677-691.

Knowles, M. (1975). Self-directed learning. Chicago: Follet.

Kolb, D. (1984). Experiential learning. Englewood Cliffs, NJ: Prentice Hall.

Markus, H. R., \& Nurius, P. (1986). Possible selves. American Psychologist, 41, 954-969.

Markus, H. R., \& Wurf, E. (1987). The dynamic self-concept: A social psychological perspective. Annual Review of Psychology, 38, 299-337.

Marsick, V., \& Watkins, K. (1990). Informal and incidental learning in the workplace. London: Routledge.

Maurer, T., Leheta, D., \& Conklin, T. (2017). An exploration of differences in content and processes underlying reflection on challenging work-related experiences. Human Resource Development Quarterly, 28, 337-368.

Maurer, T. \& Lippstreu, M., \& Hartnell, C. (2017). A model of leadership motivations, error management culture, leadership capacity, and career success. Journal of Occupational and Organizational Psychology, 90, 481-507.

McCall, M. W. (2010). Recasting leadership development. Industrial and Organizational Psychology, 3, 3-19.

McCauley, C., Ruderman, M., Ohlot, P., \& Morrow, J. (1994). Assessing the developmental components of managerial jobs. Journal of Applied Psychology, 79, 544-560.

Mumford, T. V., Campion, M. A., \& Morgeson, F. P. (2007). The leadership skills strataplex: Leadership skill requirements across organizational levels. The Leadership Quarterly, 18, 154-166.

Noe, R., Wilk, S., Mullen, E., \& Wanek, J. (1997). Employee development: Issues in construct definition and investigation of antecedents. In J. K. Ford (Ed.), Improving training effectiveness in work organizations: 153-189. Mahwah, NJ: Erlbaum. 


\section{DEVELOPMENTAL REFLECTION DIFFERENCES}

Olivares, O. J., Peterson, G., \& Hess, K. P. (2007). An existential-phenomenological framework for understanding leadership development. Leadership \& Organization Development Journal, 28, 7691.

Oreg, O. (2003). Resistance to change: Developing an individual differences measure. Journal of Applied Psychology, 88, 680-693.

Oyserman, D., \& Markus, H. R. (1990). Possible selves and delinquency. Journal of Personality and Social Psychology, 59, 112-125.

Quinton, S., \& Smallbone, T. (2010). Feeding forward: using feedback to promote student reflection and learning - a teaching model. Innovations in Education and Teaching International, 47, 125-135.

Rogers, C. R. (1969). Freedom to learn. Columbus, OH: Merrill.

Salter, M., \& Klein, G. (2007). After action reviews: Current observations and recommendations. Arlington, VA: U.S. Army Research Institute Research Report.

Schmidt, A. M., \& Ford, J. K. (2003). Learning within a learner control training environment: The interactive effects of goal orientation and metacognitive instruction on learning outcomes. Personnel Psychology, 56, 405-429.

Schon, D. (1983). The reflective practitioner: How professionals think in action. New York: Basic Books. Seibert, K. W. (1999). Reflection in action: Tools for cultivating on the job learning conditions. Organizational Dynamics, 27, 54-65.

Seibert, S. E., Kraimer, M. L., \& Liden, R. C. (2001). A social capital theory of career success. Academy of Management Journal, 44, 219-38.

Smith, E. R., \& DeCoster, J. (2000). Dual-process models in social and cognitive psychology: Conceptual integration and links to underlying memory systems. Personality and Social Psychology Review, 4, 108-131. 


\section{DEVELOPMENTAL REFLECTION DIFFERENCES}

Smith, P. (2001). Action learning and reflective practice in project environments that are related to leadership development. Management Learning, 32, 31-48.

Spector, P. E. (1988). Development of the work locus of control scale. Journal of Occupational Psychology, 61, 335-340.

Strack, F., \& Deutsch, R. (2004). Reflective and impulsive determinants of social behavior. Personality and Social Psychology Review, 8, 220-247.

Strauss, K., Griffin, M. A., \& Parker, S. K. (2012). Future work selves: How salient hoped-for identities motivate proactive career behaviors. Journal of Applied Psychology, 97, 580-598.

Trapnell, P. D., \& Campbell, J. D. (1999). Private self-consciousness and the five-factor model of personality: Distinguishing rumination from reflection. Journal of Personality and Social Psychology, 76, 284-304.

Vandewalle, D., \& Cummings, L. L. (1997). A test of the influence of goal orientation on the feedback-seeking process. Journal of Applied Psychology, 82, 390-400.

van Dyck, C., Frese, M., Baer, M., \& Sonnentag, S. (2005). Organizational error management culture and its impact on performance: A two-study replication. Journal of Applied Psychology, 90, 12281240.

Walach, H., Buchheld, N., Buttenmüller, V., Kleinknecht, N., \& Schmidt, S. (2006). Measuring mindfulness - the Freiburg mindfulness inventory (FMI). Personality and Individual Differences, 40, 1543-1555.

Zaccaro, S. J., Mumford, M. D., Connelly, M. S., Marks, M. A., \& Gilbert, J. A. (2000). Assessment of leader problem-solving capabilities. The Leadership Quarterly, 11, 37-64. 
Table 1

Descriptives and Intercorrelations among Sample 1 Variables

\begin{tabular}{|c|c|c|c|c|c|c|c|c|c|c|c|c|c|c|c|}
\hline & $\mathrm{M}$ & SD & 1 & 2 & 3 & 4 & 5 & 6 & 7 & 8 & 9 & 10 & 11 & 12 & 13 \\
\hline 1. Age & 40.23 & 9.37 & & & & & & & & & & & & & \\
\hline 2. Gender & 1.42 & 0.50 & .29 & & & & & & & & & & & & \\
\hline 3. Minority & 0.30 & 0.46 & -.13 & -.04 & & & & & & & & & & & \\
\hline 5. $\mathrm{CA}$ & 3.86 & 0.64 & -.10 & -.04 & .09 & -.03 & & & & & & & & & \\
\hline 6. DAI & 3.80 & 0.68 & -.04 & -.07 & .14 & -.05 & .72 & & & & & & & & \\
\hline 7. Need for Cognition & 3.50 & 0.63 & .28 & .19 & -.15 & .05 & .26 & .26 & & & & & & & \\
\hline 10. Reflection - PC & 4.67 & 0.93 & -.08 & .04 & .08 & -.03 & .41 & .45 & .66 & .53 & .64 & & & & \\
\hline 11. Openness & 3.44 & 0.92 & .26 & .08 & -.13 & -.11 & .13 & .10 & .57 & .16 & .33 & .53 & & & \\
\hline 12. Implicit Theories & 4.41 & 1.62 & -.20 & -.20 & .07 & -.02 & .14 & .29 & .19 & .27 & .47 & .32 & .04 & & \\
\hline 13. Work Locus of Control & 4.23 & 1.15 & -.28 & -.19 & -.01 & .13 & .30 & .39 & .34 & .38 & .53 & .45 & -.01 & .60 & \\
\hline 14. Resistance to Change & 4.26 & 1.18 & -.33 & -.20 & .11 & .14 & .26 & .39 & .29 & .29 & .64 & .46 & .14 & .70 & .65 \\
\hline
\end{tabular}

Note: $\mathrm{N}=444$. Correlations above .10 in magnitude significant at $\mathrm{p}<.05$; correlations above .13 significant at $\mathrm{p}<.01$. Gender was coded as $1=$ male, $2=$ female. Minority was coded as $0=$ non-minority, $1=$ minority. Education was coded as a range from $1=$ some high-school to $6=P h D, M D$, or other advanced degree beyond a masters. 
DEVELOPMENTAL REFLECTION DIFFERENCES

Table 2

Descriptives and Intercorrelations among Sample 2 Variables

\begin{tabular}{|c|c|c|c|c|c|c|c|c|c|c|c|c|c|}
\hline & Variable & M & SD & 1 & 2 & 3 & 4 & 5 & 6 & 7 & 8 & 9 & 10 \\
\hline 1. & Age & 36.57 & 8.19 & & & & & & & & & & \\
\hline 2. & Gender & 1.38 & 0.49 & .11 & & & & & & & & & \\
\hline 3. & Minority & 0.22 & 0.42 & -.10 & .02 & & & & & & & & \\
\hline 4. & Education & 4.21 & 0.80 & -.05 & -.09 & .04 & & & & & & & \\
\hline 5. & $\mathrm{CA}$ & 3.94 & 0.61 & -.09 & .02 & .10 & .09 & & & & & & \\
\hline 6. & DAI & 3.90 & 0.61 & -.19 & -.04 & .12 & .08 & .79 & & & & & \\
\hline 7. & Need for Cognition & 3.28 & 0.54 & .02 & .02 & .02 & .03 & .11 & .15 & & & & \\
\hline 8. & Mindfulness & 2.86 & 0.55 & -.08 & -.04 & .11 & -.06 & .37 & .39 & .20 & & & \\
\hline 9. & Rumination - PC & 4.58 & 0.88 & -.26 & -.02 & .07 & .08 & .45 & .48 & .29 & .19 & & \\
\hline 10. & Reflection - PC & 4.54 & 0.75 & -.15 & -.12 & .11 & .03 & .39 & .43 & .55 & .26 & .66 & \\
\hline 11. & Openness & 3.17 & 0.93 & .03 & -.12 & .15 & -.08 & .12 & .08 & .35 & -.02 & .19 & .47 \\
\hline 12 & IMP & 4.66 & 1.50 & -.27 & -.10 & .05 & .11 & .26 & .32 & .01 & .21 & .46 & .29 \\
\hline 13 & Work Locus of Control & 4.18 & 0.90 & -.17 & .05 & .04 & .08 & .22 & .29 & .37 & .16 & .44 & .38 \\
\hline 14 & Resistance to Change & 4.45 & 1.06 & -.25 & -.01 & -.05 & .15 & .25 & .31 & .13 & .16 & .62 & .33 \\
\hline 15 & Job Challenge Profile & 3.34 & 0.78 & -.34 & -.18 & .04 & .18 & .46 & .55 & .11 & .28 & .55 & .38 \\
\hline 16 & Error Management Culture & 3.97 & 0.52 & .04 & .07 & .06 & .03 & .53 & .51 & .20 & .44 & .24 & .29 \\
\hline 17 & Learning Goal Orientation & 5.42 & 0.91 & .04 & .07 & .06 & .03 & .53 & .51 & .20 & .44 & .24 & .37 \\
\hline 18 & Supervisor Rated Development & 5.55 & 1.27 & .08 & -.01 & .17 & .04 & .57 & .55 & .32 & .45 & .27 & .43 \\
\hline 19 & Supervisor Rated Performance & 7.05 & 1.20 & -.08 & .02 & .09 & .13 & .48 & .52 & .31 & .36 & .39 & .34 \\
\hline 20 & Career Success & 1.11 & 0.78 & -.04 & -.04 & .04 & .07 & .51 & .46 & .13 & .31 & .33 & .20 \\
\hline
\end{tabular}

Note: $\mathrm{N}=419$. Correlations above .10 in magnitude significant at $\mathrm{p}<.05$; correlations above .13 significant at $\mathrm{p}<.01$. Gender was coded as $1=$ male, 2 female. Minority was coded as $0=$ non-minority, $1=$ minority. Education was coded as a range from $1=$ some high-school to $6=P h D, M D$, or other advanced degree beyond a masters. 
Table 2

Continued

\begin{tabular}{llllllllll}
\hline Variable & 11 & 12 & 13 & 14 & 15 & 16 & 17 & 18 & 19 \\
\hline
\end{tabular}

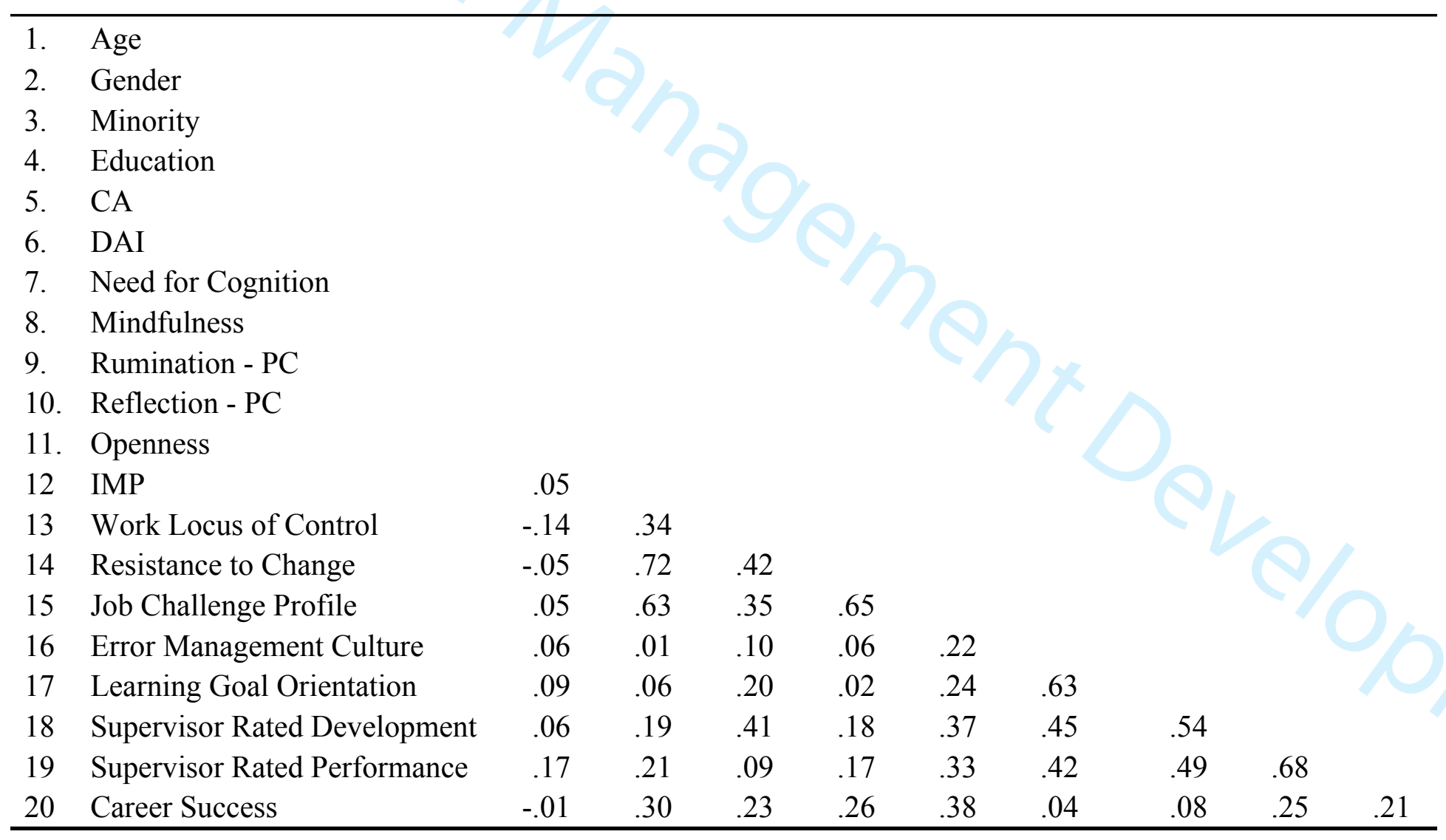


DEVELOPMENTAL REFLECTION DIFFERENCES

Table 3

\section{Path Model}

\begin{tabular}{|c|c|c|c|c|c|c|c|c|c|c|}
\hline & & & & & Deve & pment & Pron & tions & Perfo & nance \\
\hline & $\mathrm{b}$ & $\mathrm{t}$ & $\mathrm{b}$ & $\mathrm{t}$ & $\mathrm{b}$ & $\mathrm{t}$ & $\mathrm{b}$ & $\mathrm{t}$ & $\mathrm{b}$ & $\mathrm{t}$ \\
\hline \multicolumn{11}{|l|}{ Demographic Controls } \\
\hline Age & .00 & -.48 & -.01 & -1.93 & .00 & .13 & $-.02^{* *}$ & -5.71 & .01 & .87 \\
\hline Gender & .09 & 1.86 & -.01 & -.30 & .10 & .93 & -.06 & -.79 & -.14 & -1.55 \\
\hline Minority Status & .03 & .45 & .03 & .67 & .08 & .64 & .06 & .63 & -.11 & -1.05 \\
\hline Education & .01 & .39 & -.02 & -1.04 & $.14^{*}$ & 2.22 & .07 & 1.44 & -.04 & -.61 \\
\hline \multicolumn{11}{|l|}{$\underline{\text { Predictors }}$} \\
\hline Error Management Culture & $.25^{* *}$ & 7.24 & $.17^{* *}$ & 6.25 & & & & & & \\
\hline Job Challenge Profile & $.22^{* *}$ & 6.43 & $.08^{* *}$ & 3.39 & & & & & & \\
\hline Learning Goal Orientation & $.30^{* *}$ & 4.55 & $.12^{*}$ & 2.02 & & & & & & \\
\hline $\mathrm{CA}$ & & & $.57^{* *}$ & 10.61 & .36 & 1.83 & & & & \\
\hline DAI & & & & & $.80^{* *}$ & 4.02 & $.15^{*}$ & 2.25 & $.30^{* *}$ & 2.55 \\
\hline Development & & & & & & & $.10^{* *}$ & 2.79 & $.58^{* *}$ & 10.83 \\
\hline
\end{tabular}

Note: $\mathrm{N}=419$ subordinate-supervisor dyads. All coefficients unstandardized. 
Table 4

Summary of Indirect Effects

\begin{tabular}{|c|c|c|c|c|c|c|c|c|c|c|c|c|}
\hline & \multicolumn{3}{|c|}{$\begin{array}{c}\text { Developmental Action } \\
\text { Implications }\end{array}$} & \multicolumn{3}{|c|}{$\begin{array}{c}\text { Supervisor-Rated } \\
\text { Development }\end{array}$} & \multicolumn{3}{|c|}{$\begin{array}{c}\text { Career } \\
\text { Success }\end{array}$} & \multicolumn{3}{|c|}{$\begin{array}{c}\text { Supervisor-Rated } \\
\text { Performance }\end{array}$} \\
\hline & Est. & $\mathrm{t}$ & $\mathrm{CI}_{95 \%}$ & Est. & $\mathrm{t}$ & $\mathrm{CI}_{95 \%}$ & Est. & $\mathrm{t}$ & $\mathrm{CI}_{95 \%}$ & Est. & $\mathrm{t}$ & $\mathrm{CI}_{95 \%}$ \\
\hline Job Challenge Profile & $.14^{* *}$ & 6.02 & $(.10, .20)$ & $.34^{* *}$ & 7.29 & $(.25, .44)$ & & 3.77 & $(.04, .13)$ & & 7.28 & \\
\hline Learning Goal Orientation & $.13^{* *}$ & 5.34 & $(.08, .18)$ & $.24^{* *}$ & 5.81 & $(.17, .33)$ & $.06^{* *}$ & 3.68 & $(.03, .09)$ & $.20^{* *}$ & 5.48 & $(.14, .28)$ \\
\hline Error Management Culture & $.17^{* *}$ & 4.21 & $(.10, .26)$ & $.34^{* *}$ & 4.17 & $(.19, .26)$ & $.08^{* *}$ & 3.31 & $(.04, .13)$ & $.28^{* *}$ & 4.05 & $(.16, .43)$ \\
\hline Causal Analysis & & & & $.45^{* *}$ & 4.33 & $(.25, .66)$ & $.16^{* *}$ & 4.03 & $(.09, .25)$ & $.64^{* *}$ & 6.46 & $(.44, .84)$ \\
\hline Developmental Action & & & & & & & $.08^{*}$ & 2.15 & $(.22, .16)$ & $.46^{* *}$ & 4.12 & $(.25, .70)$ \\
\hline
\end{tabular}

Note: $\mathrm{N}=419$. Total indirect effects shown. All estimates unstandardized. $\mathrm{CI}_{95 \%}$ refers to the $95 \%$ confidence interval for the indirect effect. 
Figure 1. Proposed Model and Path Results

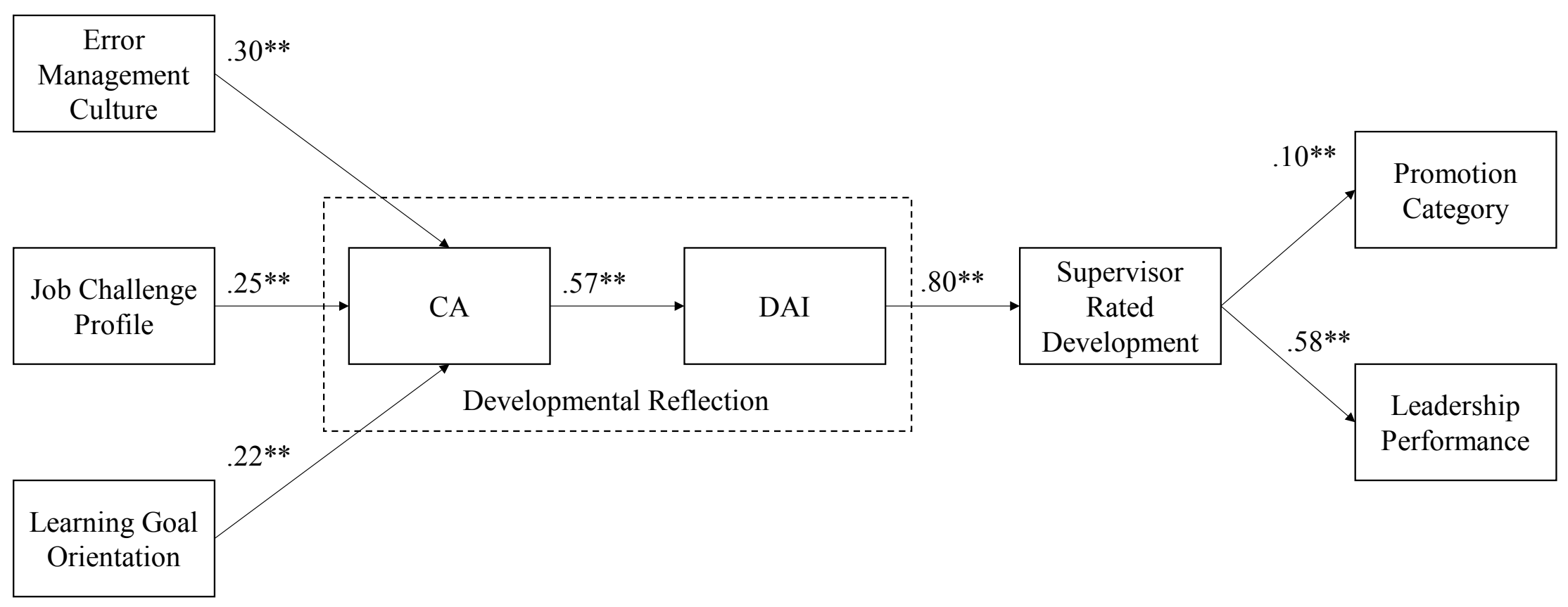

Note: $\mathrm{N}=419$. Model contains controls for demographics (age, gender, minority status, and education) not shown. Adjacent paths were estimated from antecedent variables to DAI; from CA to Development; and from DAI to Performance and Promotion (summarized in Table 3). $\chi^{2}=120.93$, df. $=11$; RMSEA $=.155 ; \mathrm{CFI}=.92 ; \mathrm{SRMR}=.06$ 


\section{Appendix: Measurement Scale Details and Analyses}

\section{Predictor Variables}

Degree of job challenge. The Development Challenge Profile (DCP; McCauley et al., 1994; $\alpha=.97$ ) was used to assess the degree of developmental job challenge. Challenges captured included stakes, scope, pressure, new and inherited problems, and unfamiliarity.

Organizational error management culture. This scale, developed by van Dyck et al. (2005; $\alpha=$ .83) asks participants to describe how their organization handles errors.

Learning Goal Orientation (LGO). LGO was captured using Vandewalle and Cummins’ $(1997 ; \alpha=.84)$ scale. This is the most frequently used scale used for this purpose, and assesses learning orientation using five items.

\section{Reflection Differences and Comparator Scales}

Causal Analysis (CA). The first dimension of reflection is a tendency to engage in causal analysis of challenging experiences in terms of factors that are under one's direct control and that are malleable to some extent, such as behavior, motivation or effort, knowledge, skills, and disposition or emotion-related constructs. To assess typical or ongoing reflection tendencies, participants were asked to indicate how much they usually thought about a factor being a cause of how developmental/challenging experiences went for them. Instructions included: "Please describe how much you have usually thought about each of the following as being a cause of how challenging experiences went for you in the past." The items were rated using a five-point scale with verbal anchors at $1=$ Little or no thinking about it being a cause; $3=$ Some thinking about it being a cause; $5=$ Extensive and detailed thinking about it being a cause. There were fifteen items spanning diverse areas of work behavior: effort, knowledge, skills, behavior, and emotions Sample items included: "My behavior"; "How hard I was trying"; "My knowledge"; "What I had the skill to do", and, "Emotions I experienced." 
Developmental Action Implications (DAI). The second dimension of reflection was a tendency to identify future action implications that flow from identifying the causal factors. As such, participants were asked to rate the extent to which, based upon their thinking about causes of experiences, they then usually plan to change, improve or enhance those underlying causes: "Based upon my thinking about causes of experiences, the extent to which I then usually plan to change, improve or enhance..." They rated the same set of items from causal analysis spanning diverse areas of work behavior (effort, knowledge, skills, behavior, and emotions) using a five-point scale with verbal anchors at $1=$ Little or none; $3=$ Some; $5=$ Extensive.

Comparator Scales. Regarding a tendency to think about oneself and one's circumstances, need for cognition was measured using the 18-item scale from Cacioppo, Petty, and Kao $(1984 ; \alpha=.87$ for Sample 1; $\alpha=.81$ for Sample 2). Reflection and rumination of private self-consciousness were each measured using a 12-item scale developed by Trapnell and Campbell (1999; reflection $\alpha=.85$ for Sample $1 ; \alpha=.75$ for Sample 2; rumination $\alpha=.89$ for Sample $1 ; \alpha=.83$ for Sample 2). Finally, mindfulness was measured using the 14item version of the Freiburg Mindfulness Inventory (Walach, Buchheld, Buttenmüller, Kleinknecht, \& Schmidt, 2006; $\alpha=.89$ for Sample 1 and .90 for Sample 2).

Regarding being open to the possibility of change and influence in one's experiences, openness was measured with a 4-item scale from the International Personality Item Pool (2001; see also Lim \& Ployhart, $2006 ; \alpha=.72$ for Sample 2, .73 for Sample 2). Resistance to change was measured with a 17-item scale (Oreg, 2003; $\alpha=.94$ for Sample 1, .93 for Sample 2). The three-item measure of implicit theory of ability (Dweck, Chiu, \& Hong, 1995; $\alpha=.91$ for both Samples) was used. Finally, we utilized Spector's (1988; $\alpha=$ .91 for Sample 1, .83 for Sample 2) 16-item work locus of control scale.

\section{Development and Career Outcomes}

Development - Supervisor rated. To measure subordinate development, supervisors rated the extent to which the person developed and improved on a variety of relevant skill dimensions over the prior year 
using a 7-point Likert scale (1 = has not improved at all on this skill to $7=$ improved an extremely great amount on this skill). To include a broad range of relevant skill content, we included items similar to that used by DeRue and Wellman (2009), drawn from Mumford, Campion, and Morgeson's (2007) taxonomy of 20 leadership skills, which was originally adapted from the Occupational Information Network $\left(\mathrm{O}^{*} \mathrm{NET}\right)$ and has been validated across organizational levels. This taxonomy includes a variety of specific, generally applicable leadership-related skills across. The coefficient alpha for this scale was .97 .

Performance - Supervisor rated. To measure subordinate performance with a special focus on leadership, we utilized the relevant scale developed by Maurer, Lippstreu, and Hartnell $(2017 ; \alpha=.94)$. Those authors asked respondents to rate targets over the prior year on leadership. Each item (e.g. "Past leadership behavior") was assessed using 9-point response scales ( $1=$ demonstrated absolute worst leadership behavior I have ever seen to $9=$ demonstrated the absolute best leadership behavior I have ever seen).

Career success-Promotions. Promotions were measured as in prior careers research (c.f. Seibert, Kraimer, \& Liden, 2001) by asking respondents to indicate the total number of promotions they received in the past year. This is "any increases in level and/or any significant increases in job responsibilities or job scope."

\section{Analyses of Reflection Construct}

We carefully analyzed the reflection construct factor structure and its distinctiveness from other related (comparator) constructs, as well as the reliability of these measures. We fit a two factor CFA for the reflection construct. This CFA considered causal analysis (CA) and developmental action implication (DAI) as separate factors that were allowed to covary. Because parallel sub-dimensions and items existed across the two CA and DAI factors (e.g. both had effort, behavior, knowledge, skill and affect subfactors and items), the model also allowed subfactors and items to covary across the CA and DAI factors. This two factor model in which CA and DAI were separate factors was compared with a CFA in which both CA and DAI loaded on a single factor. This allowed a test of whether the two processes (CA and DA) focusing on the same items were 
in fact distinct. In terms of the factor structure of the reflection scales in which we conducted CFA analyses that compared the two higher-order factors (CA and DAI) with one where the CA and DAI items were both made to load on a single factor, the proposed structure showed better fit to the data than the single factor alternative, increasing our confidence in reflection being composed of two distinct factors. Coefficients alpha for the CA measure were .92 for both Sample 1 and Sample 2, while coefficients alpha for the DA scale were .93 for Sample 1 and .91 for Sample 2.

To establish the uniqueness of the scales in comparison to existing constructs, we then introduced the comparator measures (one at a time), and ran a series of three-factor models containing our proposed two factors and the comparator variable. These three-factor models were compared to a series of two-factor models in which the comparator variables loaded on the same factor as either the CA or the DAI factor. A better fit on behalf of the proposed variables would indicate that the reflection measure is, indeed, distinct from the comparator variables. With respect to the series of three and two factor analyses to test the distinctiveness of the two factor measure was made to load on the same factor as a comparator, in all cases both the CA and DAI variables were distinct from the comparator variables; the fit of the model to the data decreased when these two variables (comparator and one of the reflection dimensions) were made to load on the same factor as the comparators. The overall fit of the model was poor for the Work Locus of Control and Need for Cognition variables (with both poor CFI and RMSEA scores); however, as our overall model fit the data well, we concluded that this was an issue with the comparator scales rather than the reflection scales. Nonetheless, the tests show that a model in which the reflection variables are distinct from the comparator variables has better fit. Results of these analyses are summarized in the Appendix Table below. 
Appendix (cont)

CFAs Contrasting CA and DAI from Each Other from Similar Variables

\begin{tabular}{|c|c|c|c|c|c|c|}
\hline 8 & $\chi^{2}$ & $\mathrm{df}$ & SRMR & CFI & RMSEA & $\mathrm{AIC}$ \\
\hline \multicolumn{7}{|l|}{$D A I$ and $C A$} \\
\hline 2 Factor Model & 941.00 & 374 & 0.060 & 0.932 & 0.049 & 26139.53 \\
\hline 1 Factor Model & 1300.18 & 375 & 0.077 & 0.888 & 0.067 & 26496.71 \\
\hline \multicolumn{7}{|l|}{ Work Locus of Control, DAI and CA } \\
\hline 3 Factor Model & 4471.14 & 956 & 0.094 & 0.752 & 0.130 & 49578.28 \\
\hline 2 Factor Model; CA collapsed & 5895.61 & 959 & 0.111 & 0.652 & 0.221 & 50996.75 \\
\hline 2 Factor Model; DAI collapsed & 6141.40 & 959 & 0.114 & 0.634 & 0.225 & 51242.55 \\
\hline \multicolumn{7}{|l|}{ Reflection Private Self, DAI and CA } \\
\hline 3 Factor Model & 2276.47 & 786 & 0.068 & 0.859 & 0.071 & 41960.88 \\
\hline 2 Factor Model; CA collapsed & 3014.14 & 789 & 0.083 & 0.789 & 0.114 & 42692.55 \\
\hline 2 Factor Model; DAI collapsed & 3039.68 & 789 & 0.083 & 0.787 & 0.094 & 42718.09 \\
\hline \multicolumn{7}{|c|}{ Rumination Private Self, DAI and CA } \\
\hline 3 Factor Model & 1751.45 & 786 & 0.054 & 0.910 & 0.061 & 41616.57 \\
\hline 2 Factor Model; CA collapsed & 2600.15 & 789 & 0.074 & 0.830 & 0.138 & 42459.27 \\
\hline 2 Factor Model; DAI collapsed & 2713.67 & 789 & 0.077 & 0.820 & 0.130 & 42572.79 \\
\hline \multicolumn{7}{|l|}{ Mindfulness, $D A I$ and $C A$} \\
\hline 3 Factor Model & 1857.44 & 869 & 0.053 & 0.906 & 0.054 & 37191.44 \\
\hline 2 Factor Model; CA collapsed & 2572.49 & 872 & 0.069 & 0.838 & 0.133 & 37900.50 \\
\hline 2 Factor Model; DAI collapsed & 2704.42 & 872 & 0.072 & 0.825 & 0.126 & 38032.43 \\
\hline \multicolumn{7}{|l|}{ Implicit Theories, DAI and CA } \\
\hline 3 Factor Model & 1130.19 & 462 & 0.059 & 0.929 & 0.060 & 30182.95 \\
\hline 2 Factor Model; CA collapsed & 2063.95 & 465 & 0.090 & 0.829 & 0.081 & 31110.71 \\
\hline 2 Factor Model; DAI collapsed & 1995.28 & 465 & 0.088 & 0.836 & 0.077 & 31042.03 \\
\hline \multicolumn{7}{|l|}{ Need for Cognition, DAI and CA } \\
\hline 3 Factor Model & 3016.02 & 1047 & 0.068 & 0.825 & 0.118 & 45579.82 \\
\hline 2 Factor Model; CA collapsed & 3993.15 & 1050 & 0.083 & 0.739 & 0.213 & 46550.95 \\
\hline 2 Factor Model; DAI collapsed & 4229.69 & 1050 & 0.086 & 0.718 & 0.221 & 46787.49 \\
\hline \multicolumn{7}{|l|}{ Resistance to Change, DAI and CA } \\
\hline 3 Factor Model & 2501.05 & 1001 & 0.060 & 0.883 & 0.074 & 48391.70 \\
\hline 2 Factor Model; CA collapsed & 3446.19 & 1004 & 0.077 & 0.809 & 0.181 & 49330.84 \\
\hline 2 Factor Model; DAI collapsed & 3607.55 & 1004 & 0.079 & 0.797 & 0.184 & 49492.20 \\
\hline \multicolumn{7}{|l|}{ Openness, DAI and CA } \\
\hline 3 Factor Model & 1117.03 & 494 & 0.055 & 0.929 & 0.064 & 30822.92 \\
\hline 2 Factor Model; CA collapsed & 2138.33 & 497 & 0.089 & 0.813 & 0.265 & 31838.22 \\
\hline 2 Factor Model; DAI collapsed & 2392.57 & 497 & 0.096 & 0.784 & 0.279 & 32092.46 \\
\hline
\end{tabular}

